\title{
Inter-laminar Strain Energy Continuity in Orthotropic Face Sandwich and Composite Plate Analysis Using Improved Higher- Order Theory
}

\author{
${ }^{1,2}$ Kasa ,Temesgen Takele $\mathrm{a}^{\mathrm{a}^{*}}$ \\ ${ }^{1}$ Addis Ababa science and Technology University, \\ Department of Electro-Mechanical and Manufacturing \\ Engineering, Addis Ababa, Ethiopia \\ ${ }^{2}$ Department of Mechanical Engineering, Pukyong \\ National University, Busan-south Korea ${ }^{\text {a }}$ \\ ttkh1@pukyong.ac.kr
}

\begin{abstract}
The main goal of this paper is, to suggest improved higher order refined theory to the analysis of perfectly bonded stack sandwich and composite laminates with usual type lamination configurations. The analysis incorporates continuous flexural and in-plane displacements in the interface. Furthermore, the transverse shear stress is continuous and also constrained with the Lagrange multiplier technique by introducing new fourteen unknown variables. The unknown variables expressed in terms of interfacial strain energy; assuming the interfacial strain energy is continuous throughout the thickness of the laminate. To determine the newly introduced flexural and in-plane unknowns' variables, total potential energy (TPE) is minimized using varational calculus. The numerical results are compared with existing reliable published papers. In general, the aforementioned approach is sufficient enough to analyze sandwich and laminate structures with the required accuracy.
\end{abstract}

Keywords- Refined theory; Flexural displacement; In-plane displacement; Constraint; Strain Energy; TPE

\section{INTRODUCTION}

Nowadays, light weight sandwich and composite structures are widely used in the aeronautical/aerospace and marine industries to gain superior safety, higher payload and good fatigue resistance property. To achieve the above appropriate properties, multilayered laminate structure should requires advanced understanding of statics and dynamical behavior of composite materials[1]. Likewise, sandwich and composite structures are complicated in analysis and design aspect; because some unforeseen failure modes like delamination's are considered [2-4].To overcome the aforementioned challenges inter-laminar strain energy continuity assumption based refined modeling has suggested. This approach gives limitless ways to design and optimize the laminates in accordance with the applied external load.
In general, there is no exact mathematical assumption to analyze sandwich and laminated composite plate with the required accuracy. To plug in the gap, this paper play a vital role by considering layer by layer refined theory analytical approach with assumption of perfect bond between layers and the interfacial strain energy continuity throughout the thickness of the laminate.

The energy in the interfaces to estimate the mechanical behavior of the laminate structures. The theory is implemented on layer by layer technique; in which total potential energy of each layer has minimized by Lagrange multiplier, in-plane and flexural displacement using varaitional calculus. To maintain the principle of continuous uniform deformation theory the plies are bounded perfectly and the continuity conditions constrained by the Lagrange multipliers to satisfy the boundary conditions in terms of in-plane and flexural displacements. Using the above approach, the in plane and flexural stresses can be easily analyzed in each layer of the lamina [5-7].

In general, we considered a refined composite and sandwich plate theory that incorporates continuous strain energy within the interfaces to calculate the mechanical behavior of laminate structures. Our theory is implemented using a layer-by-layer technique in which the total potential energy of each layer is minimized via the Lagrange multiplier to obtain in-plane and flexural displacements using variational calculus. To maintain the principles behind the continuous uniform deformation theory, the plies are perfectly bonded and the continuity conditions are constrained by the Lagrange multipliers to satisfy the governing equilibrium equation using boundary conditions to determine the unknown variables. By applying this approach, the flexural and in-plane stresses are easily analyzed at each layer of the lamina. Further, these formulations are calculated to yield easier plate configurations in two dimensions. 


\section{POTENTIAL ENERGY MINIMIZATION}

In order to determine the natural boundary conditions and governing equilibrium equations in flexural and in-plane form, we can use minimization of total potential energy. In ord er to analyze the above geometry of plate we have been made $t$ he following fundamental assumptions.(1) The laminate have uniform thickness and symmetrical at the mid-plane; to simplif $\mathrm{y}$ the governing equilibrium equations; (2)The core is compres sible; (3) The global axes does not coincide with the local axes of symmetry; (4) The skin plies bonds to core perfectly; (5) Th e core and skin layers are linear elastic (6) The face and core 1 ayers are considered as 2D and 3D structures respectively; (7) The plate assumed to be flat and naturally it has no curve. Under this assumption, all odd polynomial superscript coeffici ents become zero in the equilibrium equations. Therefore, the nine governing equilibrium equations of the laminate can be se parated into five flexural and four in-plane components. By incorporating the constraints, the total potential energy minimized as follows.

$$
\begin{array}{lll}
\frac{\partial\left(\Pi+U_{\text {int }}\right)}{\partial u_{i}^{o}}=0 & , & \frac{\partial\left(\Pi+U_{\text {int }}\right)}{\partial \phi_{i}}=0 \\
\frac{\partial\left(\Pi+U_{\text {int }}\right)}{\partial \psi_{i}}=0 & , & \frac{\partial\left(\Pi+U_{\text {int }}\right)}{\partial \Lambda_{u i}^{a}}=0 \\
\frac{\partial\left(\Pi+U_{\text {int }}\right)}{\partial \theta_{i}}=0 & , & \frac{\partial\left(\Pi+U_{\text {int }}\right)}{\partial \Lambda_{i j}^{a}}=0
\end{array}
$$

The interfacial strain energy formulated as follows

$$
U_{\text {int }}=\frac{1}{2} \int_{A_{\text {int }}}\left(\begin{array}{l}
\left(\Lambda_{u_{1}}^{T o p} u_{1}^{(1)}-\Lambda_{u_{1}}^{T o p} u_{1}^{(2)}\right) \\
+\left(\Lambda_{u_{2}}^{T o p} u_{2}^{(1)}-\Lambda_{u_{2}}^{T o p} u_{2}^{(2)}\right) \\
+\left(\Lambda_{u_{3}}^{T o p} u_{3}^{(1)}-\Lambda_{u_{3}}^{T o p} u_{3}^{(2)}\right) \\
+\left(\Lambda_{13}^{C T} \tau_{13}^{(2)}-\Lambda_{13}^{C T} \tau_{13}^{(3)}\right) \\
+\left(\Lambda_{23}^{C T} \tau_{23}^{(2)}-\Lambda_{23}^{C T} \tau_{23}^{(3)}\right) \\
+\left(\Lambda_{13}^{T} \tau_{13}^{(3)}+\Lambda_{23}^{(1)}-\Lambda_{23}^{B o t} \tau_{u_{1}}^{(3)} u_{1}^{(2)}\right) \\
+\left(\Lambda_{u_{2}}^{B o t} u_{2}^{(1)}-\Lambda_{u_{2}}^{B o t} u_{2}^{(2)}\right) \\
+\left(\Lambda_{u_{3}}^{B o t} u_{3}^{(1)}-\Lambda_{u_{3}}^{B o t} u_{3}^{(2)}\right) \\
+\left(\Lambda_{13}^{B C} \tau_{13}^{(1)}-\Lambda_{13}^{B C} \tau_{13}^{(2)}\right) \\
+\left(\Lambda_{23}^{B C} \tau_{23}^{(1)}-\Lambda_{23}^{B C} \tau_{23}^{(2)}\right) \\
+\left(\Lambda_{13}^{B} \tau_{13}^{(1)}+\Lambda_{23}^{B} \tau_{23}^{(2)}\right)
\end{array}\right) d x_{1} d x_{2}
$$

\section{ANALYTICAL CLOSED FORM SOLUTIONS}

The aim of analytical closed form solutions is, to determine the unknown variables analytically. The unknown variables can be obtained from the displacement and interlaminar strain energy continuity formulas. For this approach the Lagrange, in-plane and flexural displacements equations are expanded as Fourier series based on boundary conditions. Considering a load:

$$
q=Q_{m n}^{o} \sin \alpha x_{1} \sin \beta x_{2}
$$

\section{RESULTS}

Symmetric three layered simply supported square composite and sandwich plate with height is equal to $h=10 t$ be used. Applying various aspect $\operatorname{ratios}(\mathrm{S})$ the numerical results of flexural displacement, inter-laminar shear stress and in plane stress are shown in the Table-1 and 2.Table-1 2 and 3 shows that, the percentage error obtained from the suggested approach is in a good agreement with exact elasticity solution[8] and in conclusion while the error percentages decreasing as the plate aspect ratio increase. For $\mathrm{S}$, equal to 4 , 10 and 20, the new approach gives a better estimate of inplane stress and inter-laminar shear stress than other approaches for the given aspect ratios. Furthermore, for very thin ply ( $\mathrm{a} / \mathrm{h}=50$ and above) the suggested theory contributes a better accuracy for in-plane stresses as compared to other classical models.

The results in the tables report are obtained by the following non-dimensional formulas:

$$
\begin{aligned}
& \bar{u}_{1}=u_{1}\left(\frac{100 * h^{3} E_{22}}{q a^{4}}\right), \bar{u}_{2}=u_{2}\left(\frac{100 * h^{3} E_{22}}{q a^{4}}\right), \\
& \bar{\sigma}_{22}=\sigma_{22}\left(\frac{h^{2}}{q a^{2}}\right) \\
& \bar{u}_{3}=u_{3}\left(\frac{100 * h^{3} E_{22}}{q a^{4}}\right), \bar{\sigma}_{11}=\sigma_{11}\left(\frac{h^{2}}{q a^{2}}\right) \bar{\tau}_{12}=\tau_{12}\left(\frac{h^{2}}{q a^{2}}\right),
\end{aligned}
$$

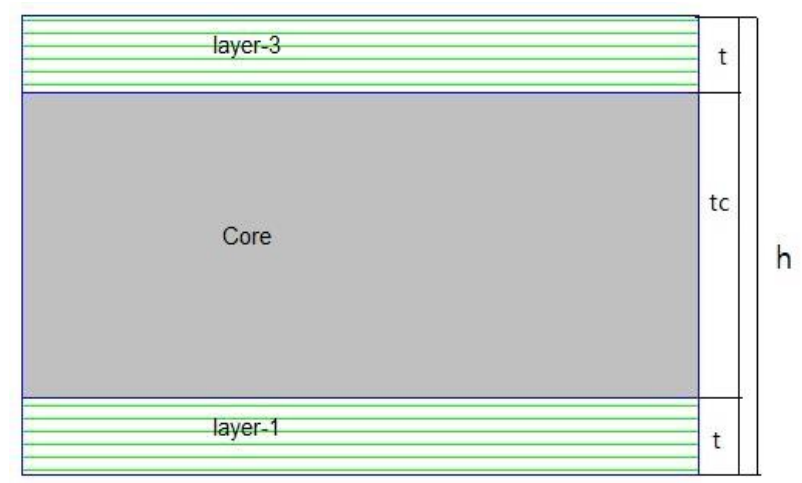

Figure-1. Sandwich plate 
Table: 1 Error Percentage of maximum stress in nondimensional form $(0 /$ core $/ 0)$

\begin{tabular}{c|ccccr}
\hline $\mathrm{S}$ & source & $\bar{\sigma}_{11}$ & $\bar{\sigma}_{22}$ & \multicolumn{1}{c}{$\bar{\tau}_{12}$} & \multicolumn{1}{c}{$\bar{u}_{3}$} \\
$\mathbf{2}$ & Elasticity[8] & \pm 2.653 & \pm 0.3919 & \pm 0.2338 & 0.1402 \\
& New & \pm 2.6593 & \pm 0.3917 & \pm 0.2337 & 0.1405 \\
& Error & $\mathbf{0 . 2 3 7 5}$ & $\mathbf{0 . 0 5 1 0 3}$ & $\mathbf{0 . 0 4 2 7 7}$ & $\mathbf{0 . 0 7 1 3 3}$ \\
$\mathbf{4}$ & Elasticity[8] & \pm 1.512 & \pm 0.2533 & \pm 0.1481 & 0.1072 \\
& New & \pm 1.5139 & \pm 0.25314 & \pm 0.1479 & 0.1075 \\
& Error & $\mathbf{0 . 1 2 5 7}$ & $\mathbf{0 . 0 6 3 1 6 6}$ & $\mathbf{0 . 0 2 0 5 7}$ & $\mathbf{0 . 2 7 8 9}$ \\
$\mathbf{1 0}$ & Elasticity[8] & \pm 1.152 & \pm 0.1099 & \pm 0.0707 & 0.0527 \\
& New & \pm 1.153 & \pm 0.10976 & \pm 0.07059 & 0.0529 \\
$\mathbf{2 0}$ & Error & $\mathbf{0 . 0 8 6 8}$ & $\mathbf{0 . 0 3 6 3 9}$ & $\mathbf{0 . 1 5 5 5 8}$ & $\mathbf{0 . 3 7 9 5}$ \\
& Elasticity[8] & \pm 1.110 & \pm 0.070 & \pm 0.0511 & 0.0361 \\
& New & \pm 1.112 & \pm 0.070017 & \pm 0.05118 & 0.0364 \\
$\mathbf{5 0}$ & Error & $\mathbf{0 . 0 1 8 0 1}$ & $\mathbf{0 . 0 1 4 2}$ & $\mathbf{0 . 1 5 2 4}$ & $\mathbf{0 . 5 2 8}$ \\
& Elasticity[8] & \pm 1.099 & \pm 0.0569 & \pm 0.0446 & 0.0306 \\
& New & \pm 1.0991 & \pm 0.056905 & \pm 0.04463 & 0.03064 \\
$\mathbf{1 0 0}$ & Error & $\mathbf{0 . 0 1 8}$ & $\mathbf{0 . 0 0 8 7 8}$ & $\mathbf{0 . 0 8 2 6}$ & $\mathbf{0 . 6 1 5}$ \\
& Elasticity[8] & \pm 1.098 & \pm 0.0550 & \pm 0.0437 & 0.0297 \\
& New & \pm 1.099 & \pm 0.0551 & \pm 0.0438 & 0.02982 \\
& Error & $\mathbf{0 . 0 1 5}$ & $\mathbf{0 . 0 0 5 9}$ & $\mathbf{0 . 0 7 6}$ & $\mathbf{0 . 7 4 0 3}$
\end{tabular}

Table-2:Error percentage for orthotropic face sandwich

\begin{tabular}{|c|c|c|c|c|c|}
\hline$S$ & source & $\bar{\sigma}_{11}$ & $\bar{\sigma}_{22}$ & $\bar{\tau}_{12}$ & $\bar{u}_{3}$ \\
\hline \multirow[t]{3}{*}{2} & Kant -2[5] & \pm 4.0665 & \pm 0.531 & \pm 0.5184 & 39.0218 \\
\hline & New & \pm 4.0668 & \pm 0.5340 & \pm 0.5171 & 39.042 \\
\hline & Error & 0.0074 & 0.0073 & 0.2505 & 0.0518 \\
\hline \multirow[t]{3}{*}{4} & Kant -2[5] & \pm 1.7931 & \pm 0.2128 & \pm 0.2702 & 14.4949 \\
\hline & New & \pm 1.7704 & \pm 0.2138 & \pm 0.2725 & 14.471 \\
\hline & Error & 5.79 & 5.46 & 4.76 & 6.0985 \\
\hline \multirow[t]{3}{*}{10} & Kant -2[5] & \pm 0.8344 & \pm 0.1527 & \pm 0.1352 & 3.8899 \\
\hline & New & \pm 0.8345 & \pm 0.1533 & \pm 0.1354 & 3.8925 \\
\hline & Error & 0.0046 & 0.052 & 0.1479 & 0.0668 \\
\hline \multirow[t]{3}{*}{100} & Kant -2[5] & \pm 0.6647 & \pm 0.0642 & \pm 0.0699 & 1.0806 \\
\hline & New & \pm 0.6648 & \pm 0.0641 & \pm 0.0700 & 1.0827 \\
\hline & Error & 0.015 & 0.1558 & 0.0121 & 0.628 \\
\hline
\end{tabular}

Table-3 Error percentage for composite plate

\begin{tabular}{|c|c|c|c|c|c|}
\hline $\mathbf{s}$ & source & $\bar{\sigma}_{11}$ & $\bar{\sigma}_{22}$ & $\bar{\tau}_{12}$ & $\bar{u}_{3}$ \\
\hline \multirow[t]{3}{*}{2} & Elasticity[8] & 0.937 & 0.669 & 0.0859 & 4.9362 \\
\hline & New & 0.93814 & 0.670096 & 0.086099 & 4.93853 \\
\hline & Error & 0.12166 & 0.16442 & 0.2328 & 0.0474 \\
\hline \multirow[t]{3}{*}{4} & Elasticity[8] & 0.755 & 0.556 & 0.0505 & 1.7287 \\
\hline & New & 0.7558698 & 0.556799 & 0.0505627 & 1.72907 \\
\hline & Error & 0.1152 & 0.14388 & 0.12475 & 0.021735 \\
\hline \multirow[t]{3}{*}{10} & Elasticity[8] & 0.59 & 0.285 & 0.0289 & \\
\hline & New & 0.590631 & 0.285359 & 0.028932 & 0.7171 \\
\hline & Error & 0.1069 & 0.1263 & 0.1136 & \\
\hline \multirow[t]{3}{*}{20} & Elasticity[8] & 0.552 & 0.21 & 0.0289 & \\
\hline & New & 0.552563 & 0.210242 & 0.028930 & 0.5427 \\
\hline & Error & 0.1021 & 0.1154 & 0.1045 & \\
\hline \multirow[t]{3}{*}{50} & Elasticity[8] & 0.541 & 0.185 & 0.0216 & 0.4432 \\
\hline & New & 0.541471 & 0.185172 & 0.0216209 & 0.443282 \\
\hline & Error & 0.087 & 0.093 & 0.0968 & 0.0187 \\
\hline \multirow[t]{3}{*}{100} & Elasticity[8] & 0.539 & 0.181 & 0.0213 & \\
\hline & New & 0.539442 & 0.181164 & 0.021319 & 0.4347 \\
\hline & Error & 0.082 & 0.0906 & 0.0932 & \\
\hline
\end{tabular}

\section{SUMMARY}

In this paper, we explored and analyzed the accuracy of a layer-by-layer refined theory model with flexural and in-plane displacement continuity in a laminate composite and sandwich plate. The improved higher-order theory was applied for efficient execution of the refined theory. Inter-laminar continuity equations were also constrained using Lagrange multipliers by introducing new variables. The existing and newly introduced variables were then solved through a total potential energy minimization technique. Here, Navier-type analytical closed-form solutions were adopted for analyses. Considering simply supported boundary conditions, we analyzed higher and lower-aspect ratio sandwich plates. Further, the sandwich plate was subjected to sinusoidal distributed loading on the top face. Given this, we calculated all flexural displacements, in-plane displacements, in-plane stress, and interfacial shear stresses and then compared them with exact values presented in previous studies. Further, we performed some parametric tests, with results showing that the aspect ratio increases the accuracy of the analysis also increases for the above approach. We also found that each displacement or stress component requires its own plate model (which differs according to the change in outputs) to obtain exact results. Further, the accuracy of the solution also depends on thickness coordinate $x_{3}$. The key advantage of our proposed approach is the ability to obtain remarkably accurate results for all ranges of aspect and modular ratios. Further, if $\mathrm{n}$ eeded, it is possible to enhance our proposed approach by deco mposing the lower-aspect ratio layer into a number of higher a spect ratio layers.

\section{ACKNOWLEDGMENT}

I acknowledge NIIDE and Pukyong National University for their material support of this research.

\section{REFERENCES}

[1] J. R. Vinson, The behavior of sandwich structures of isotropic and composite materials: CRC Press, 1999.

[2] A. C. Garg, "Delamination - a damage mode in composite structures," Engineering Fracture Mechanics, vol. 29, no. 5, pp. 557-584, 1988.

[3] F. Crossman, W. Warren, A. Wang, and G. Law Jr, "Initiation and growth of transverse cracks and edge delamination in composite laminates Part 2. Experimental correlation," Journal of Composite Materials, vol. 14, no. 1, pp. 88-108, 1980. 
[4] P. P. Camanho, and C. G. Dávila, "Mixed-mode decohesion finite elements for the simulation of delamination in composite materials," 2002.

[5] T. Kant, and K. Swaminathan, "Analytical solutions for the static analysis of laminated composite and sandwich plates based on a higher order refined theory," Composite structures, vol. 56, no. 4, pp. 329-344, 2002.

[6] T. Kant, and K. Swaminathan, "Estimation of transverse/interlaminar stresses in laminated composites-a selective review and survey of current developments," Composite structures, vol. 49, no. 1, pp. 65-75, 2000.

[7] J. N. Reddy, "A simple higher-order theory for laminated composite plates," Journal of applied mechanics, vol. 51, no. 4, pp. 745-752, 1984.

[8] N. J. Pagano, "Exact solutions for rectangular bidirectional composites and sandwich plates," Mechanics of composite materials, pp. 86-101: Springer, 1994. 